\title{
How to succeed as a junior doctor 䇺
}

\author{
Author: Michael Azad ${ }^{\mathrm{A}}$
}

The Royal College of Physicians (RCP) organised an inaugural 1-day conference, the aim of which was to look into some of the key issues that junior doctors need to address when beginning their careers. The target audience was medical students but a few foundation doctors were also in attendance. The conference also helped to increase RCP engagement with medical students and foundation doctors. The day was co-chaired by Professor Kate Thomas (vice dean, University of Birmingham) and Dr Andrew Macleod and Dr Kanwaljit Sandhu (RCP regional advisors for the West Midlands).

KEYWORDS: Career, ePortfolio, junior doctor, medical student, succeed

\section{Introduction}

The transition from being a medical student to life as a junior doctor can be daunting. The Royal College of Physicians (RCP) recognises this and thus developed a pioneering 1-day regional conference in conjunction with the University of Birmingham Medical School. The aim of this conference was to explore some of the issues faced by junior doctors in the first stages of their training and to equip medical students and foundation year doctors with some of the basic knowledge and skills needed to deal with them. The breadth of topics covered was impressive and certainly covered many of the key issues that needed to be addressed (Box 1). In fact, the topics were chosen after a survey of local foundation doctors making them highly pertinent.

\section{The ePortfolio and personal development plan}

The ePortfolio is the electronic companion that follows a doctor throughout their time as a trainee in medicine. It is a record of professional development, ultimately used as part of the Annual Review of Competence Progression (ARCP). Trainees must demonstrate competence in pre-defined areas of the set curriculum before progressing to the next stage of training. The use of workplace-based assessments (WPBAs) as a means of establishing evidence of required competencies by linking them to the curriculum was explained. Junior doctors should contemplate their short-term, intermediate and long-term

Author: ${ }^{A}$ specialty registrar in geriatric medicine and general internal medicine, Queen's Medical Centre, Nottingham University Hospitals, Nottingham, UK career goals and these should form the basis of their personal development plan (PDP). The PDP should be discussed with the educational supervisor and regularly reviewed at appraisal meetings between the trainee and supervisor. Specific learning objectives and the evidence needed to show that they have been met should be identified. Learning objectives can be based on areas of interest, including placement-specific skills, quality improvement work, research or teaching, and they should be centred on the individual trainee's needs. Goals should be specific, measurable, achievable, relevant and time-limited (think SMART). The ePortfolio may seem like a complicated training tool to a junior doctor but it should not become a distraction from an individual's career advancement; if used well, it can facilitate seamless progression from one stage of training to the next.

\section{Quality improvement}

Quality improvement can be defined as achieving better patient outcomes and experience through changing provider behaviour and organisation through using a systematic change method and strategies. ${ }^{1}$ This session aimed to introduce the concept of quality improvement and stress its importance in clinical practice. Quality improvement endeavours should start with a specific aim. Then, by means of process mapping and driver diagrams (which were both explained with examples), one can arrive at an area of practice to implement a change. The regular collection of accurate data related to process and outcome measures is essential for a successful project. Examples of data presented in a time-series fashion were displayed and such data can be powerful drivers for further improvement and change. One commonly used and structured approach to quality improvement was discussed: plan-do-study-act (PDSA) cycles. ${ }^{2}$ Audit was cited as a specific example of quality

\section{Box 1. Key issues addressed}

The ePortfolio and personal development plan (PDP)

Quality improvement

Research and publications

Common dilemmas

Disciplinary skills to be an effective leader

Professional links within primary care

Tips for the first month 
improvement measured against standardised practices of care. Quality improvement work can help with career progression, development of leadership skills and give insight into the workings of healthcare systems, all of which are important as a junior doctor.

\section{Research and publications}

Research involves hypothesis testing by data gathering, ultimately culminating in advancement of knowledge. Hypotheses related to relevant and important clinical issues are usually more powerful, especially in areas of research with unanswered but answerable questions. Completion of some form of good research work can be invaluable in enhancing a junior doctor's curriculum vitae. It is also important for appraisal purposes and demonstrating evidence for curriculum competencies. Some of the perceived barriers to undertaking research include lack of funding, supervision or time.

Publications can take many forms, such as case reports, original articles, review articles or letters to the editor. Authorship of work should be clarified at the earliest opportunity and one should seek an experienced supervisor. Consideration should be made to how the work may be clinically translatable. General advice included showing eagerness to participate in research and actively seeking out research opportunities.

\section{Common dilemmas}

The speaker (Dr Daryl Leung) acknowledged the challenge of covering the topics of bettering bad news, dealing with relatives, and complaints and medico-legal issues in his 30 minute slot. When delivering bad news, the importance of non-verbal as well as verbal communication was emphasised. The context of the situation in relation to the patient, relatives and/or carers should be contemplated. Family members must be considered in the care of all patients in order for the patient's care to be truly holistic. Developing a rapport with family members is essential when dealing with patients who lack mental capacity and best interests decisions are needed to be made.

Complaints from patients and/or relatives can be written or verbal, formal or informal. The process from involvement of the Patient Advice and Liaison Service (PALS) to complaint meetings and, rarely, intervention from the Parliamentary and Health Service Ombudsman was described. The key role of the coroner in selected cases is to identify the cause of death and not to apportion blame. Junior doctors may face many difficult dilemmas in their careers but many of these situations could be avoided by regular and effective communication with patients and their families. Support and advice can be sought from consultants and other senior healthcare professionals as well as medico-legal defence unions. Such difficult situations usually offer the opportunity for reflective practice and learning.

\section{Disciplinary skills to be an effective leader}

Junior doctors should know their limitations and when and how to seek support. This can be sought from healthcare professionals other than doctors, where relevant. Junior doctors may feel anxious before night shifts but this can be ameliorated by paying close attention to behaviours prior to and during shift working, including ensuring adequate rest and eating well. On-call shifts should begin with a clear handover process where doctors finishing a shift can direct the incoming team's attention to specific tasks and unwell patients in the area they cover. If there is risk of imminent deterioration in patients who are unwell, help should be sought early. In other less critical situations, the necessary information should be gathered before considering what procedures or investigations may be needed next. Thought should be given to a provisional diagnosis and management plan and then discussed with a more senior doctor.

There is a wide variety of jobs that a junior doctor may be asked to do while on-call, such as performing procedures, preparing discharge letters or reviewing patient results. It is vital that junior doctors are organised and able to prioritise tasks. Documentation in patient records should be clear, thorough and legible. Being on-call may seem disconcerting but the experience should be viewed as an extremely rich learning opportunity.

\section{Professional links within primary care}

A good working relationship between primary and secondary care is essential for high quality patient care. GPs have a wealth of knowledge and expertise that secondary care doctors can utilise. They can provide useful collateral histories as well as execute and follow through post discharge management plans. Discharge documents should be concise and accurate. Medication changes should be clear and the reasons for these changes specified. Reasonable tasks for the GP to carry out or follow up should be cited explicitly with respective timescales for completion. Abbreviations should be avoided where possible. If there is urgent information that needs to be conveyed to a GP, then a junior doctor should not shy away from contacting the GP directly by telephone. Whether one works in primary care or secondary care, we should all strive to work together in partnership for the benefit of patients.

\section{Tips for the first month}

Dr Chris Huntley gave useful and generic advice for new junior doctors. When starting a new job, induction week can be a barrage of all kinds of information. The speaker had taken the time to collate various helpful tips for junior doctors from patients, nurses, trainees and consultants. These are summarised in Box 2.

\section{Box 2. Tips for the first month}
Introduce yourself
Listen to nurses' concerns on the ward
Explain management plans to patients and relatives
Be organised
Remember to take a break
Organise annual leave sooner rather than later
Be patient
Engage with relatives
Be enthusiastic 


\section{How to look cool on the post-take ward round}

The final session overlapped in content with the previous one and reiterated some of the advice already mentioned. Additional tips included being punctual and dressing smartly. Hospital trusts usually have a vast array of local guidelines and these should be utilised when necessary. Junior doctors should make themselves noticed but for the right reasons and they should aim to impress on the post-take ward round by making astute diagnoses, for example.

\section{Conflicts of interest}

The author declares no conflicts of interest.

\section{References}

1 Vaux E, Went S, Norris M, Ingham J. Learning to make a difference: introducing quality improvement methods to core medical trainees. Clin Med 2012;12:520-5.

2 The King's Fund. Model for improvement. London: The King's Fund. Available online at www.kingsfund.org.uk/projects/pfcc/ model-improvement [Accessed 24 June 2016].

Address for correspondence: Dr M Azad, Queen's Medical Centre, Nottingham University Hospitals, Derby Road, Nottingham NG7 2UH, UK.

Email:michael.azad@nhs.net

\section{Assessing trainees in the workplace An e-learning module for secondary care doctors}

Three hours of CPD-approved interactive learning covering:

$>$ feedback

> supervised learning events (SLES)

$>$ workplace-based assessments (WPBAs)

$>$ the role of the Annual Review of Competence Progression (ARCP).

For more information please visit: www.rcplondon.ac.uk/elearning

\section{Royal College} of Physicians

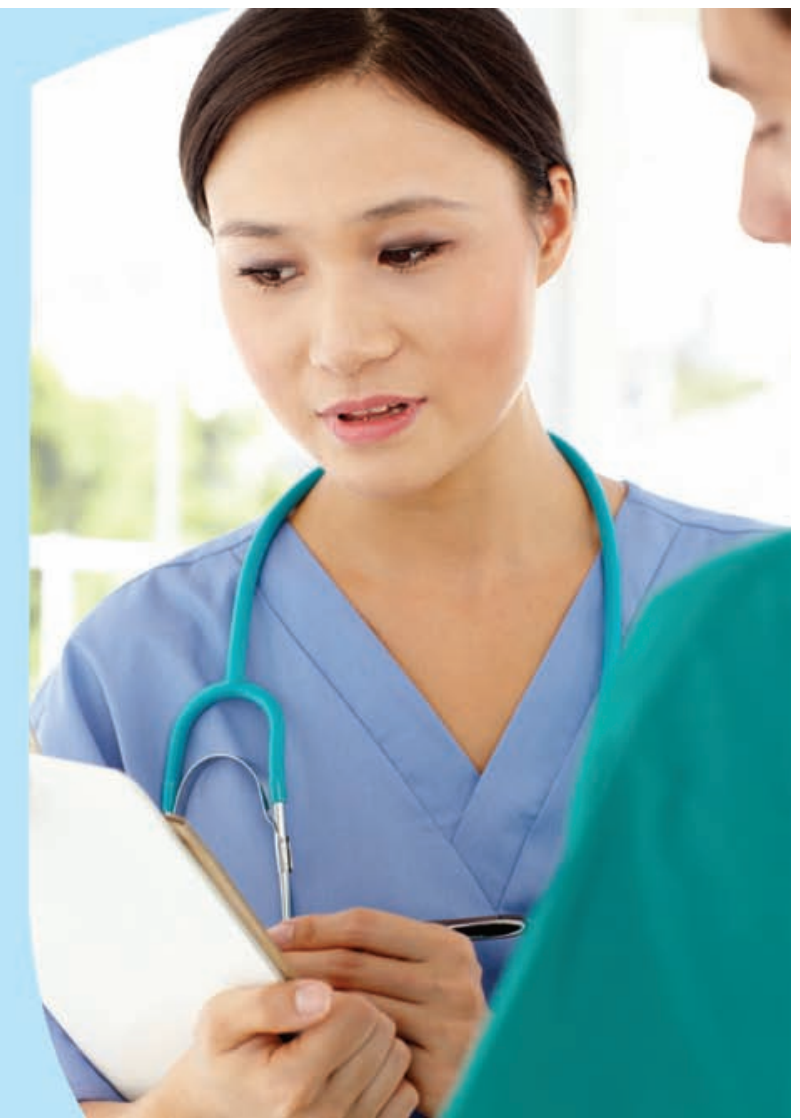

